Accepted for Publication

Water Resources Research

February, 2003

\title{
A Particle Tracking Approach to Simulating Transport in a Complex Fracture
}

\author{
Chin-Fu Tsang and Christine Doughty \\ Earth Sciences Division \\ E.O. Lawrence Berkeley National Laboratory \\ Berkeley, California 94720, USA
}

\begin{abstract}
Nearly all of the studies of flow and transport in fractured rocks have assumed that each fracture can be modeled as an open space between two surfaces with constant or variable separation. Field observations of rock fractures have shown that a fracture in the field can be rather more complex. Recent studies indicate that a complex fracture can be characterized as a thin fracture zone having several interconnected sub-fractures, which can contain mechanically dislodged and chemically altered materials with an enhanced porosity. The present paper proposes a particle-tracking approach to calculate solute transport in a complex fracture, with structures in the fracture thickness normal to the fracture plane. These structures include sub-fractures, dead-end pores, gouge materials, small matrix blocks, and the adjacent rock matrix, all providing material in which solute diffusion and sorption can occur. The method is described; an example is provided to show its feasibility and the reasonableness of its parameter dependence; and, finally, direction for its further development is discussed.
\end{abstract}

Keywords: 5104 Fracture and flow, 5139 Transport properties, 1829 Groundwater hydrology 


\section{Introduction}

Flow and transport in a single fracture in low-permeability crystalline rocks has been widely studied over the past decade and more (Cvetkovic et al., 1999; Moreno et al., 1990; Nordqvist et al., 1992; Tsang and Tsang, 1989; Brown et al., 1998; Moreno and Tsang, 1994; and others). In nearly all of these studies, the single fracture is modeled as an open space between two surfaces with a constant or variable separation. This separation or aperture is bounded by rock matrix, which plays a significant role in solute transport in the fracture through the process of matrix diffusion and sorption (Neretnieks, 1980; Rasmuson and Neretnieks, 1986).

Recent studies of rock fractures have shown that a natural fracture can be rather complex. Sharp et al. (1996) and Robinson et al. (1998) reviewed field observations on a fracture skin region where porosity and permeability can be quite different from those of the rock matrix. Bossart and Mazurek's investigation (1991) of the structural geology of a shear fracture showed that it can be considered as a thin fracture zone with a thickness of the order of one centimeter. Within this fracture zone, water is carried in several interconnected sub-fractures containing mechanically dislodged and chemically altered materials of enhanced porosity. These fractures are embedded in a fabric of granitic rock with large porosity within the fracture zone. Hadermann and Heer (1996) modeled flow and transport through such a complex fracture by conceptualizing it as a set of three to six small, parallel, uniform-aperture fractures embedded in the thickness of the complex fracture or fracture zone. Solutes moving through these sub-fractures experience diffusion and sorption into the rock materials within and outside the fracture zone.

The present paper proposes a particle-tracking method for modeling flow and transport in a complex fracture, accounting for transmissivity variability over the fracture plane and the structure in its thickness normal to this plane. Thus, the fracture thickness is characterized by the presence of several smaller sub-fractures containing gouge materials and surrounded by altered rock matrix, and bounded on either side by "semi-infinite" 
rock matrix. In the next section, a characterization of the complex fracture is given and its conceptualization discussed. The tools to be used for flow and transport calculations in such a complex fracture are then presented, together with a procedure for carrying out the computation. An example with calculational results is then given to illustrate the method. Finally a discussion of further research directions concludes the paper.

\section{Definition of a Complex Fracture and its Conceptualization}

In this paper, we shall use "complex fracture" and "fracture zone" interchangeably and refer to "sub-fractures" as smaller fractures embedded within the fracture zone. Mazurek et al. (2001) characterizes a fracture zone as having two embedded sub-fractures partially filled with gouge materials. A schematic cross section of the complex fracture is shown in Figure 1. On either side of each sub-fracture are fracture coatings and microfractures. The two sub-fractures are connected by a number of minor fractures called splays. In the example shown in Figure 1, the two sub-fractures in the fracture zone are separated by $\sim 2$ $\mathrm{cm}$. Considering flow through such a complex fracture, we can identify a number of factors that play a role. These are listed in Table 1. 
Table 1. Factors affecting flow and transport in a complex fracture

\begin{tabular}{|l|l|}
\hline Factor & Description \\
\hline a & Spatial transmissivity variations over the plane of the fracture zone \\
\hline b & $\begin{array}{l}\text { Multiple sub-fractures within the fracture zone (two sub-fractures } \\
\text { are shown in Figure 1) }\end{array}$ \\
\hline c & $\begin{array}{l}\text { Aouge materials within each of the sub-fractures } \\
\text { coating and microfractures }\end{array}$ \\
\hline d & $\begin{array}{l}\text { Splays, or minor fractures, in between the two sub-fractures; these } \\
\text { break up the rock into blocks with sizes of a fraction of the width of } \\
\text { the complex fracture }\end{array}$ \\
\hline e & $\begin{array}{l}\text { Dead-end pores and splays into neighboring rocks } \\
\text { Rock matrix on two sides of the fracture zone, which may be altered } \\
\text { in the immediate neighborhood of the fracture zone with a higher } \\
\text { porosity region }\end{array}$ \\
\hline g &
\end{tabular}

Diffusion and sorption retard tracers moving through the sub-fractures, with the magnitude of retardation depending on the nature of the rock material present (i.e., gouge, fracture skin, altered rock matrix, or unaltered rock matrix). With its large surface to volume ratio, the small blocks of gouge material provide an effective retardation mechanism, followed by intermediate-size blocks of altered rock interspersed by splays and dead-end pores, and finally the semi-infinite rock matrix on either side of the fracture zone. Fracture skins may either enhance or inhibit diffusion and sorption, depending on their physical and chemical character. As a function of time, particles move initially by advection through the system and soon are retarded by diffusion and sorption through the gouge and intermediate blocks. Then, because of their finite sizes, these blocks become saturated by the particles and provide no further retardation and diffusive effect. Thus, only the semi-infinite rock matrix, with a much smaller surface to volume ratio, allows diffusion/sorption to the particles in the longer term. 
Now let us conceptualize the problem so that a calculational method can be designed. This conceptualization depends strongly on the available information or data on the complex fracture. The proposed approach attempts to make use of all potentially available data. In addition, those features without specific information are combined where possible into lumped parameters. The values of these lumped parameters can then be obtained through calibration against tracer transport experiments.

We propose first to calculate flow in the fracture plane $(x, y)$ based on well-test data. These well tests are normally conducted with packers bracketing the thickness of the fracture zone and no detailed hydraulic resolution in the $z$ direction can be expected. In principle, we can obtain data on transmissivity variability over the fracture plane, $T(x, y)$. Given transmissivity values at a reasonable number of $(x, y)$ locations, we can construct a probability density function for a two-dimensional (2-D) $\log T$ distribution with a spatial correlation range $\lambda$. If the $\log T$ distribution turns out to be normal, then it can be simply characterized by its mean $\log (\bar{T})$ and standard deviation $\sigma_{\log T}$. Then, realizations of the transmissivity field can be generated using a geostatistical method, and the 2-D flow $\mathbf{q}(x, y)$ through each grid cell at $(x, y)$ can be calculated numerically (see, for example, Moreno et al., 1991, Tsang and Tsang, 1989; Tsang and Neretnieks, 1998). Given q(x, $y$ ), the conventional particle-tracking procedure is to calculate the residence time, $t$, in a grid block $\Delta x \Delta y$ within the 2-D flow field. The 2-D tracer travel time would then be the sum of these residence times as the tracer traverses the flow domain and emerges at the observation area. Typically, $t$ is calculated by assuming that the cubic law holds locally at each grid cell, relating $q$ to an apparent aperture $b$, i.e. $q \propto b^{3}$. In this case, a flow volume can be defined as $V=b \Delta x \Delta y$. Thus, $t=V / q \propto \Delta x \Delta y / b^{2}$.

Now, for our problem, we need to modify the 2-D residence time $t$ to account for the structures in the third dimension, normal to the fracture plane. For this purpose, we shall assume that the 2-D flow $\mathbf{q}(x, y)$ represents the sum of the flows in the sub-fractures distributed over the thickness (z-direction) of the complex fracture, and that the flow direction of each sub-fracture is the same as that of $\mathbf{q}(x, y)$. For the case of two subfractures, derivations can then be made as follows. 
We hypothesize that at each $(x, y)$ location, the two sub-fractures have flow $q_{1}$ going through one with aperture $b_{1}$ and $q_{2}$ going through the other with aperture $b_{2}$. We introduce the ratio of flow in the two sub-fractures, $q_{2} / q_{1}$ as a new parameter

$$
\alpha=\frac{q_{2}}{q_{1}} .
$$

Therefore the total flow $q$ in each grid cell at $(x, y)$ may be written as

$$
q=q_{1}+q_{2}=q_{1}(1+\alpha) .
$$

Here we have written $\mathbf{q}(x, y), \mathbf{q}_{1}(x, y)$, and $\mathbf{q}_{2}(x, y)$ simply as $q, q_{1}$, and $q_{2}$, respectively. Thus, $q_{1}$ and $q_{2}$ in terms of $q$ are given by:

$$
\begin{aligned}
& q_{1}=\frac{q}{1+\alpha} \\
& q_{2}=\frac{q}{1+\alpha^{-1}} .
\end{aligned}
$$

The sub-volumes of a grid cell at the $(x, y)$ location, corresponding to the large and small sub-fractures, respectively, are

$$
\begin{aligned}
& V_{1}=b_{1} \Delta y \Delta x \\
& V_{2}=b_{2} \Delta y \Delta x .
\end{aligned}
$$

Assuming that the cubic law is applicable locally at a grid cell $(x, y)$ in each sub-fracture, $q_{\mathrm{i}} \propto b_{i}^{3}(i=1,2)$, we have from Equation (1)

$$
\frac{b_{2}}{b_{1}}=\frac{V_{2}}{V_{1}}=\alpha^{1 / 3}
$$


To conserve flow, we require Equation (2) to hold, and since the cubic law is assumed,

$$
b^{3}=b_{1}^{3}+b_{2}^{3} .
$$

This equation implies $b \neq b_{1}+b_{2}$, and, from Equations (3), (4) and (7),

$$
\begin{aligned}
& b_{1}=\frac{b}{(1+\alpha)^{1 / 3}} \\
& b_{2}=\frac{b}{\left(1+\alpha^{-1}\right)^{1 / 3}} \\
& b_{1}+b_{2}=\frac{\left(1+\alpha^{1 / 3}\right) b}{\left(1+\alpha^{-1}\right)^{1 / 3}}
\end{aligned}
$$

which is the reason we refer to $b$ from the 2-D calculation as the apparent $b$. This is a typical situation for a fracture zone containing a number of small fractures, each obeying the cubic flow law, with the requirement that the total flow be kept constant. Using $V=$ $b \Delta y \Delta x$, it can be derived from Equations (5) through (8) that

$$
\begin{aligned}
& V_{1}=\frac{V}{(1+\alpha)^{1 / 3}} \\
& V_{2}=\frac{V}{\left(1+\alpha^{-1}\right)^{1 / 3}} .
\end{aligned}
$$

Therefore, we get residence times for the two sub-fractures, respectively, of

$$
t_{1}=\frac{V_{1}}{q_{1}}=\frac{1+\alpha}{(1+\alpha)^{1 / 3}} \frac{V}{q}=(1+\alpha)^{2 / 3} t
$$




$$
t_{2}=\frac{V_{2}}{q_{2}}=\frac{1+\alpha^{-1}}{\left(1+\alpha^{-1}\right)^{1 / 3}} \frac{V}{q}=\left(1+\alpha^{-1}\right)^{2 / 3} t
$$

These residence times are a function of the parameter $\alpha$.

For each particle, the probability of flow to be in the sub-fracture 1 is

$$
P_{1}=\frac{q_{1}}{q}=\frac{1}{1+\alpha}
$$

and the probability of flow to be in the sub-fracture 2 is

$$
P_{2}=\frac{q_{2}}{q}=\frac{1}{1+\alpha^{-1}} \text {. }
$$

As an aside, note that the weighted residence time can be shown to be

$$
P_{1} t_{1}+P_{2} t_{2}=\frac{1+\alpha^{1 / 3}}{(1+\alpha)^{1 / 3}} t=\frac{V_{1}+V_{2}}{V} t
$$

again illustrating the fact that $V_{1}+V_{2}$ is not simply $V$, but is a function of $\alpha$.

A similar development can be made for three or more sub-fractures in the complex fracture with the introduction of additional parameters like $\alpha^{\prime}$. If there are many subfractures in the complex fracture, then $\mathbf{q}(x, y)$ can be divided into a probabilistic distribution of flow in the $\mathrm{z}$ direction: $P[\mathbf{q}(z)]$, such that at each $(x, y)$, the flows at different $z$ values add up to $\mathbf{q}(x, y)$. This probabilistic function can be gaussian, bimodal, or multi-modal, and can be characterized by two or three more parameters. The spatial flow distribution in the fracture plane $\mathbf{q}(x, y)$ and its extension into the third dimension 
accounts for Factors $\mathrm{a}$ and $\mathrm{b}$ in the Table 1 list of factors characterizing the complex fracture.

Next, the presence of gouge materials in the sub-fractures (Table 1, Factor c), minor fractures and splays forming in matrix blocks between the sub-fractures (Table 1, Factor e), and dead-end pores (Table 1, Factor f) are accounted for by assuming that water flow within these objects is negligible compared with flows in sub-fractures and that they act only as materials for diffusion and sorption. Fortunately, for some field sites, direct measurements on sample materials are possible to provide the diffusivity and sorptive coefficient for solutes of interest. The rock volume on either side of the fracture zone (Table 1, Factor g) is assumed similarly to act as a semi-infinite matrix medium for solute diffusion and sorption.

We categorize the media into which diffusion and sorption occur into three types. The first two correspond to "matrix blocks" with characteristic size $2 r_{\mathrm{m}}$ : (1) small blocks with $2 r_{\mathrm{m}} \approx 10-1000 \mu \mathrm{m}$ representing fault gouge (Table 1, Factor c), and (2) intermediate-size blocks between the two sub-fractures with $2 r_{\mathrm{m}}$ of the order of $1 \mathrm{~mm}-1$ $\mathrm{cm}$, representing the effects of splays and minor fractures between the sub-fractures (Table 1, Factors e and f). The third type is a semi-infinite medium representing rock matrix on either side of the complex fracture (Table 1, Factor g). Thus, at every step of the particle movement, we can assign it to encounter one of these three diffusion/sorption types with a prescribed probability. The relative importance of advection and diffusion depends on fracture aperture, so either $b_{1}$ or $b_{2}$ is used in the diffusion/sorption calculation, depending on which sub-fracture was chosen for the particle. At the present time, skin effects (Table 1, Factor d) are not included in our model.

\section{Approach to Modeling Transport in a Complex Fracture}

Our approach is based on the particle tracking method applied to the conceptual model described above, using three computer codes. The first is a code to generate the $T(x, y)$ heterogeneous field, using a probability density function for $\log T$ and spatial correlation 
$\lambda$. The $\log T$ probability function is estimated from available transmissivity data at a number of locations over the fracture plane, and the generated field is conditioned to these data. In the example presented in the next section, we have used the SISIM code, which is part of the geostatistical library GSLIB by Deutsch and Journel (1998). The code is based on sequential indicator geostatistics, which does not require the assumption of a particular distribution such as lognormal and which, furthermore, allows different spatial correlation ranges for different ranges of $T$ values. Tsang et al. (1996) used this code to generate 3D fracture-porous rock systems. For our present application, only $T$ ( $x$, $y$ ) in $2 \mathrm{D}$ is generated with this code.

The second tool is a code to calculate $\mathbf{q}(x, y)$ over this transmissivity field. In our case, we use a simple finite difference code. Once $\mathbf{q}(x, y)$ is obtained, it is divided into flows within sub-fractures in the $\mathrm{z}$ direction $\mathbf{q}\left(x, y ; z_{i}\right)$, as discussed in the last section, with a new parameter $\alpha$ for the case of two sub-fractures. Cases with more than two subfractures or with a sub-fracture distribution can also be done straightforwardly.

Once $\mathbf{q}\left(x, y ; z_{i}\right)$ is known, a particle tracking code is used to account for matrix diffusion into finite matrix blocks or into a semi-infinite medium. The code we use for the example below is the THEMM code (Tsang and Tsang, 2001). In this method, for each grid cell, the advective residence time of a particle is first computed. Then, using a semianalytic method involving a probabilistic function, a time delay is calculated to account for diffusion and sorption into rock matrix blocks of a specified radius $r_{m}$, that are encountered by the particle. The new parameters required, besides the rock block size $2 r_{\mathrm{m}}$, are matrix block porosity $\phi_{\mathrm{m}}$, linear-sorption isotherm $K_{D}$, and solute diffusion coefficient $D_{\mathrm{e}}$ (an effective diffusion coefficient formed by the product of the solute diffusion coefficient in water, the porosity, and the tortuosity of the matrix block). As discussed in the last section, rock matrix blocks of three types will be used, so that at each time step, the particles will have a prescribed probability of encountering these three types of rock materials. Three corresponding sets of matrix diffusion and sorption parameters can be estimated from laboratory tests of samples or by calibration to tracer tests in the field. 
Thus, within the THEMM code, at every step of particle tracking $\left(x, y, z_{1}\right.$, or $\left.z_{2}\right)$, the particles encounter one of the three classes of matrix blocks with a prescribed probability. At each step, a time delay due to diffusion and sorption into these matrix types is calculated and added to the particle advective residence time. Then, particle locations as a function of time are traced over the fracture zone plane, and breakthrough curves at the outlet or observation well are computed by summing over all arriving particles.

The above calculational method is illustrated by an example presented in the next section.

\section{Example}

In this example, which takes advantage of the capability of the SISIM code, we assume that the probability density function representing a range of observed transmissivity data can be divided into six transmissivity groups (Table 2). The correlation length for the group with the highest transmissivity is taken to be $1 \mathrm{~m}$, and the correlation lengths for the other five groups are all taken to be $0.3 \mathrm{~m}$. These data are consistent with a data set from an actual field site at the Hard Rock Lab, Äspö, Sweden (Winberg et al., 2000). Figure 2 shows a transmissivity distribution generated with SISIM as well as stream lines illustrating the steady-state flow field obtained when a head difference is applied between the top and bottom of the model. The two sides are no-flow boundaries. 
Table 2. Model Properties.

\begin{tabular}{|c|c|c|c|c|c|c|}
\hline \multicolumn{7}{|l|}{ Fracture Properties } \\
\hline \multicolumn{7}{|l|}{ Transmissivity } \\
\hline Geometric mean & \multicolumn{6}{|c|}{$3.5 \mathrm{E}-8 \mathrm{~m}^{2} / \mathrm{s}$} \\
\hline Minimum & \multicolumn{6}{|c|}{$7.1 \mathrm{E}-10 \mathrm{~m}^{2} / \mathrm{s}$} \\
\hline Maximum & \multicolumn{6}{|c|}{$1.1 \mathrm{E}-5 \mathrm{~m}^{2} / \mathrm{s}$} \\
\hline \multicolumn{7}{|l|}{ SISIM code parameters } \\
\hline Transmissivity group & 1 & 2 & 3 & 4 & 5 & 6 \\
\hline Weighting & 0.27 & 0.13 & 0.20 & 0.20 & 0 & 0.20 \\
\hline $\log _{10} \mathrm{~T}$ level & -8.8 & -8.2 & -7.2 & -6.4 & -5.4 & -5.0 \\
\hline $\begin{array}{r}\text { Spatial correlation } \\
\text { lengths }\end{array}$ & $0.3 \mathrm{~m}$ & $0.3 \mathrm{~m}$ & $0.3 \mathrm{~m}$ & $0.3 \mathrm{~m}$ & $0.3 \mathrm{~m}$ & $1 \mathrm{~m}$ \\
\hline Fracture zone thickness & \multicolumn{6}{|c|}{$2 \mathrm{~cm}$} \\
\hline Matrix Properties & \multicolumn{2}{|c|}{ Small blocks } & \multicolumn{2}{|c|}{ Intermediate blocks } & \multicolumn{2}{|c|}{ Semi-infinite } \\
\hline Probability (\%) & \multicolumn{2}{|c|}{20} & \multicolumn{2}{|c|}{30} & \multicolumn{2}{|c|}{50} \\
\hline $2 r_{\mathrm{m}}$ & \multicolumn{2}{|c|}{$0.1 \mathrm{~cm}$} & \multicolumn{2}{|c|}{$1 \mathrm{~cm}$} & \multicolumn{2}{|c|}{-} \\
\hline$\phi_{\mathrm{m}}$ & \multicolumn{2}{|c|}{0.2} & \multicolumn{2}{|c|}{0.01} & \multicolumn{2}{|c|}{0.003} \\
\hline$D_{\mathrm{e}}$ & \multicolumn{2}{|c|}{ 2.E-10 $\mathrm{m}^{2} / \mathrm{s}$} & \multicolumn{2}{|c|}{ 1.E-12 $\mathrm{m}^{2} / \mathrm{s}$} & \multicolumn{2}{|c|}{$8.4 \mathrm{E}-14 \mathrm{~m}^{2} / \mathrm{s}$} \\
\hline
\end{tabular}

We assume that there are two sub-fractures within the fracture zone thickness, and consider the $\alpha$ values to range from 0 to 1 . Typical properties $\left(r_{\mathrm{m}}, \phi_{\mathrm{m}}, D_{\mathrm{e}}\right)$ for the three types of rock matrix are listed in Table 2. In our simulation, we let a non-sorbing tracer be released along a 2-m horizontal line perpendicular to the overall flow direction, $5 \mathrm{~m}$ from the up-gradient boundary of the model $(6.5<x<8.5, y=5$ in Figure 2). Tracer is collected all along the down-gradient boundary of the model $(y=15 \mathrm{~m})$, to produce one aggregate breakthrough curve. 
Figure 3 summarizes the results of transport in a complex fracture. The calculated breakthrough curves of tracer concentration are shown as a function of time for cases with diffusion into the three types of matrix blocks, with probability ratios of $0.2,0.3$, and 0.5 for the gouge, intermediate blocks and semi-infinite matrix, respectively. The sets of curves shown may be discussed in terms of two separate groups. The first group contains curves shown by lines with square symbols, labeled "single type, $\alpha=0$ ". They show the influence of individual contributions of diffusion-retardation caused by gouge, intermediate blocks, and semi-infinite matrix, respectively, by turning off the effect of the other two contributions. As compared with the advection-only curve (the broken line), diffusion into intermediate and gouge materials is shown as a shift of the curve to later times and saturation of tracers in finite rock volumes is indicated by the fact that the shape of the curves are similar to that of the advection-only case. The semi-infinite curves, on the other hand, show a small impact initially resulting from the low surface-tovolume ratio for diffusion, but then its effect persists to very large times. The solid curve, labeled with " 0 ”" (meaning $\alpha=0$ ), reflects the sum of the three effects.

The second group of curves in Figure 3 shows solid curves without symbols, which are labeled $0,0.1,0.3$, and 1 . These are cases with diffusion into all matrix types, with $\alpha=0,0.1,0.3$ and 1 , respectively. When $\alpha=0$, there is only flow through one subfracture; increasing values of $\alpha$ indicate increasing quantities of flow through the second sub-fracture, until when $\alpha=1$, the two sub-fractures have identical flow properties. Pore volume $\left(V_{1}+V_{2}\right)=\left(\mathrm{b}_{1}+\mathrm{b}_{2}\right) \Delta \mathrm{x} \Delta \mathrm{y}$ increases as $\alpha$ increases (Equation 11), so breakthrough time is delayed as $\alpha$ increases (Equation 18). For $0<\alpha<1$, the breakthrough curves show a long tail of late-arriving tracer that has spent a significant period of time in the smaller sub-fracture, with greater opportunity for matrix diffusion.

Our main results are obtained for one realization of the $2 \mathrm{D}$ heterogeneous $\log T$ field generated by the SISIM code. To evaluate the effect of multiple realizations, we generated four more realizations based on the same statistical parameters (Table 2) and re-calculated the breakthrough curves for two cases, that of gouge-only and that of $\alpha=0.1$. The spread of the results for the five realizations is indicated by the horizontal 
bars shown on these two curves. The small spread shows that in our example the impact of multiple realizations is limited.

\section{Discussions and Concluding Remarks}

An approach has been proposed and described to calculate solute transport in a complex fracture with structures in the fracture thickness normal to the fracture plane. These structures include sub-fractures, dead-end pores, gouge materials, intermediate matrix blocks, and the adjacent rock matrix medium, all of which provide means for solute diffusion and sorption. An example is provided to demonstrate the approach, and results show that the proposed method is feasible, and the parameter dependence and sensitivity are reasonable.

The present approach includes sufficient physics to account for the structure of the complex fracture, considering that its detailed geometry and properties are not available in field situations. Generally, we should introduce a minimum number of new parameters that are needed to reproduce all field data. If we assume that there are two sub-fractures in a ladder-like structure (see Figure 1), we need to introduce one additional parameter $\alpha$ to represent the relative flows through the two sub-fractures. Further, we assume the probabilities for the tracer to encounter the three types of matrix materials that participate in diffusion and adsorption. The diffusion and sorption properties can, in

principle, be obtained by laboratory measurements for, respectively, the fracture gouge materials, rock materials between the two sub-fractures, and the rock matrix neighboring the complex fracture. The parameter $\alpha$, however, can best be estimated through a calibration study against a set of tracer transport test data. Similarly, if we had introduced three sub-fractures, then we would have two parameters describing their relative flows, $\alpha$ and $\alpha^{\prime}$, and these would need to be estimated by calibration against several sets of tracer test data.

In general, the parameter $\alpha$ may vary over space, and thus its value obtained through calibration must be considered as a representative value averaged in some sense over the 
fracture plane. It is still an open issue, if the tracer migration distances in the calibration and in later prediction studies are very different, whether this average $\alpha$ value is still valid for the latter. In fact, this is part of the general problem of extrapolation of what we learn from short-term tracer tests to predictions of tracer transport over larger time and space scales.

One interesting question is what happens to the calibrated parameter values if we were to use a simple fracture model, based on the conventional advective-dispersive equation, to match the tracer breakthrough curves obtained from results for a complex fracture. In the simple fracture model, diffusion and sorption into gouge materials and intermediate blocks are ignored, and thus their effects have to be represented by the property values of the semi-infinite rock matrix. This would lead to calibrated values significantly larger than laboratory measurements of the rock matrix samples.

One of the features of a complex fracture, the fracture skin between flow in the fracture aperture and the adjacent rock matrix, has not been included in the present approach. This requires a method to calculate solute migration through several media successively, which is currently under study and development. Another related problem is the impact of the conceptual model proposed in this paper on flow and pressure distributions both in the fracture plane and in the third dimension normal to it. This deserves further study. 


\section{Acknowledgments}

Discussions with Masahiro Uchida of the Japan Nuclear Cycle Research Institute (JNC) are gratefully acknowledged. We are also appreciative of the reviews of K. Karasaki and C. Oldenburg, and those of anonymous reviewers and the Associate Editor of Water Resources Research. Their comments have greatly helped to improve the manuscript. Work is supported by JNC through a bi-national JNC/DOE Agreement between JNC and US Department of Energy (DOE), under the overview of DOE's Office of Environmental Management, Office of Science and Technology and conducted at the Ernest Orlando Lawrence Berkeley National Laboratory, under the auspices of DOE Contract No. DEAC03-76SF00098. 


\section{References}

P. Bossart and M. Mazurek, Grinmsel Test Site -Structural geology and water flow-paths in the migration shear-zone. Nagra Technical Report, NTB 91-12, Nagra, Wettingen, Switzerland, 1991.

S.R. Brown, A. Ciprihan, and R. Hardy, Experimental observations of fluid flow channels in a single fracture. J. Geophys. Res., 103 (B3), 5125-5132, 1998.

V. Cvetkovic, J-O Selroos, and H. Cheng, Transport of reactive solute in single fractures, J. Fluid Mech., 318: 335-356, 1999.

C.V. Deutsch, and A.G. Journel, GSLIB - Geostatistical Software Library and User's Guide, Oxford Univ. Press, New York, Second Edition, 1998.

J. Hadermann, W. Heer, The Grimsel (Switzerland) migration experiment: integrating field experiments, laboratory investigation and modelling, Journal of Contaminant Hydrology, 21, 87-100, 1996.

M. Mazurek, P. Bossart and J. Hermanson, Classification and characterization of waterconducting features at Äspö., Proc. Intern. Seminar First TRUE Stage, Äspö Hard Rock Laboratory, Sweden, September 11-13, 2000, published by the Swedish Nuclear Fuel and Waste Management Company (SKB), Stockholm, Sweden, 2001.

L. Moreno and C.F. Tsang, Flow channeling in strongly heterogeneous porous media: A numerical study, Water Resour. Res., 30(5), 1421-1430, 1994.

L. Moreno, Y.W. Tsang, C.F. Tsang, and I. Neretnieks, Some anomalous features of flow and solute transport arising from fracture variability, Water Resour. Res., 26(10), 23772391, 1990. 
I. Neretnieks, Diffusion in the rock matrix: An important factor in radionuclide retardation?, J. Geophys. Res., 85(B8), 4379-4397, 1980.

A.W. Nordqvist, Y.W. Tsang, C.F. Tsang, B. Dverstorp and J. Andersson, A variableaperture fracture network model for flow and transport in crystalline Rocks, Water Resour. Res., 28 (6), 1703-1713, 1992.

N. Robinson, J.M. Sharp, Jr., I. Kreisel, Contaminant transport in sets of parallel finite fractures with fracture skins, Journal of Contaminant Hydrology, 31, 83-109, 1998.

J.M. Sharp, Jr., I. Kreisel, K.L. Milliken, R.E. Mace, N.I. Robinson, Fracture skin properties and effects in solute transport. Geotechnical and environmental implications. Proceedings Second North Amer. Rock Mechnics Symposium, 2, pp. 1329-1336, Montreal, 1996a.

A. Rasmuson and I. Neretnieks, Migration of radionuclides in fissured rock: The influence of micropore diffusion and longitudinal dispersion, J. Geophys. Res., 86(B5), 3749-3758, 1981.

C.F. Tsang and I. Neretnieks, Flow channeling in heterogeneous fractured rocks. Reviews of Geophysics, 36(2), 275-298, 1998.

Y.W. Tsang and C.F. Tsang, A Particle-Tracking Method for Advective Transport in Fractures with Diffusion into Finite Matrix Blocks, Water Resources Research, 37(3): 831-835, 2001. LBNL-42634.

Y. W. Tsang and C. F. Tsang, Flow channeling in a single fracture as a two-dimensional strongly heterogeneous permeable medium. Water Resources Research, 25, (9) 20762080, 1989, LBL-26596. 
Y. W. Tsang, C. F. Tsang, F. V. Hale, and B. Dverstorp, Tracer Transport in a Stochastic Continuum Model of Fractured Media. Water Resources Research, Vol. 32, No. 10, pp. 3077-3092, 1996.

A. Winberg, P. Andersson, J. Hermanson, J. Byegard, V. Cvetkovic, and L. Birgersson, Aspo Hard Rock Laboratory, Final report of the first stage of the tracer retention understanding experiments, Swedish Nuclear Fuel and Waste Management Co., TR-0007, 2000. 


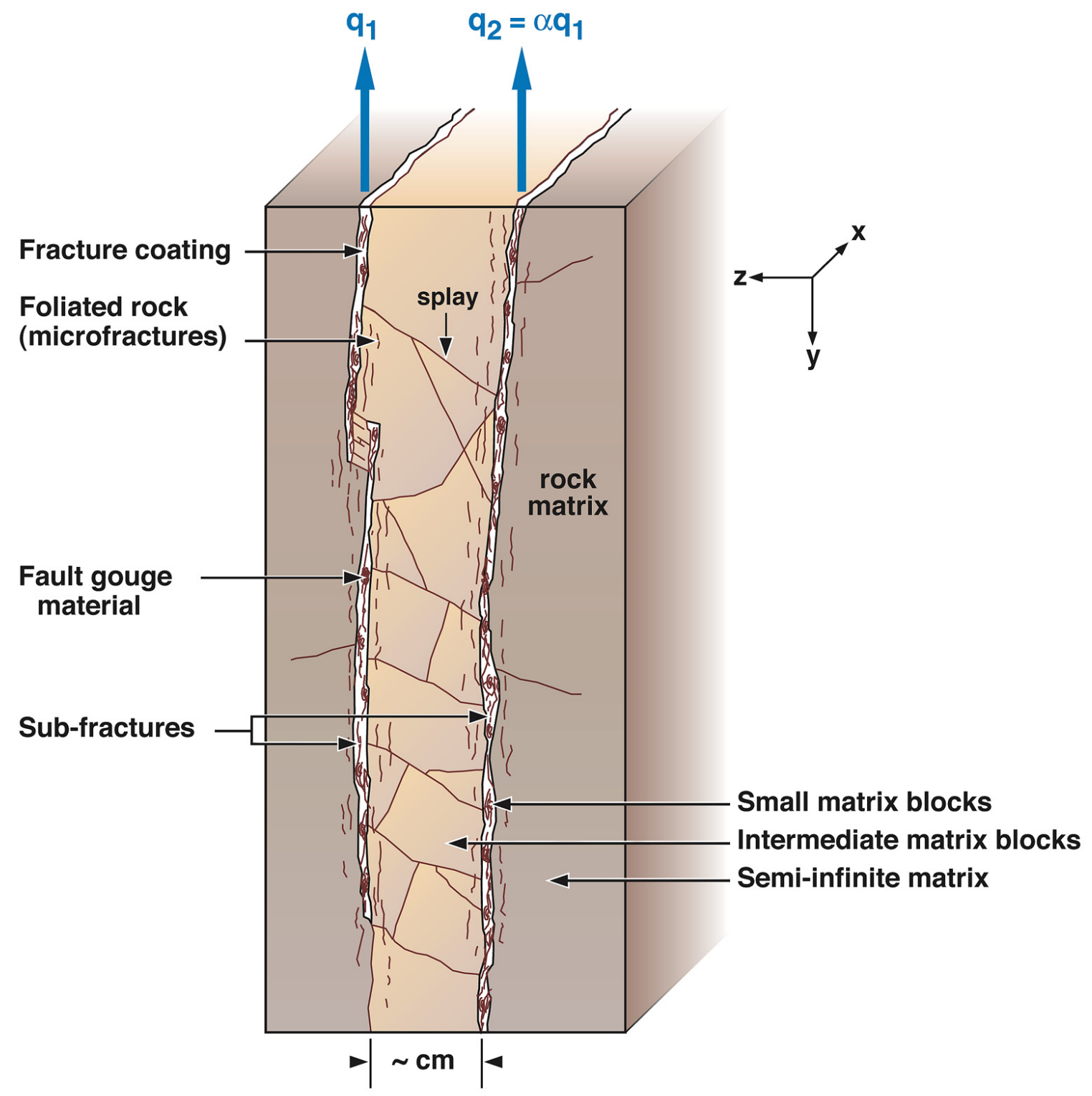

Figure 1. Structure of a complex fracture or master fault (adapted from Mazurek et al., 2001). 


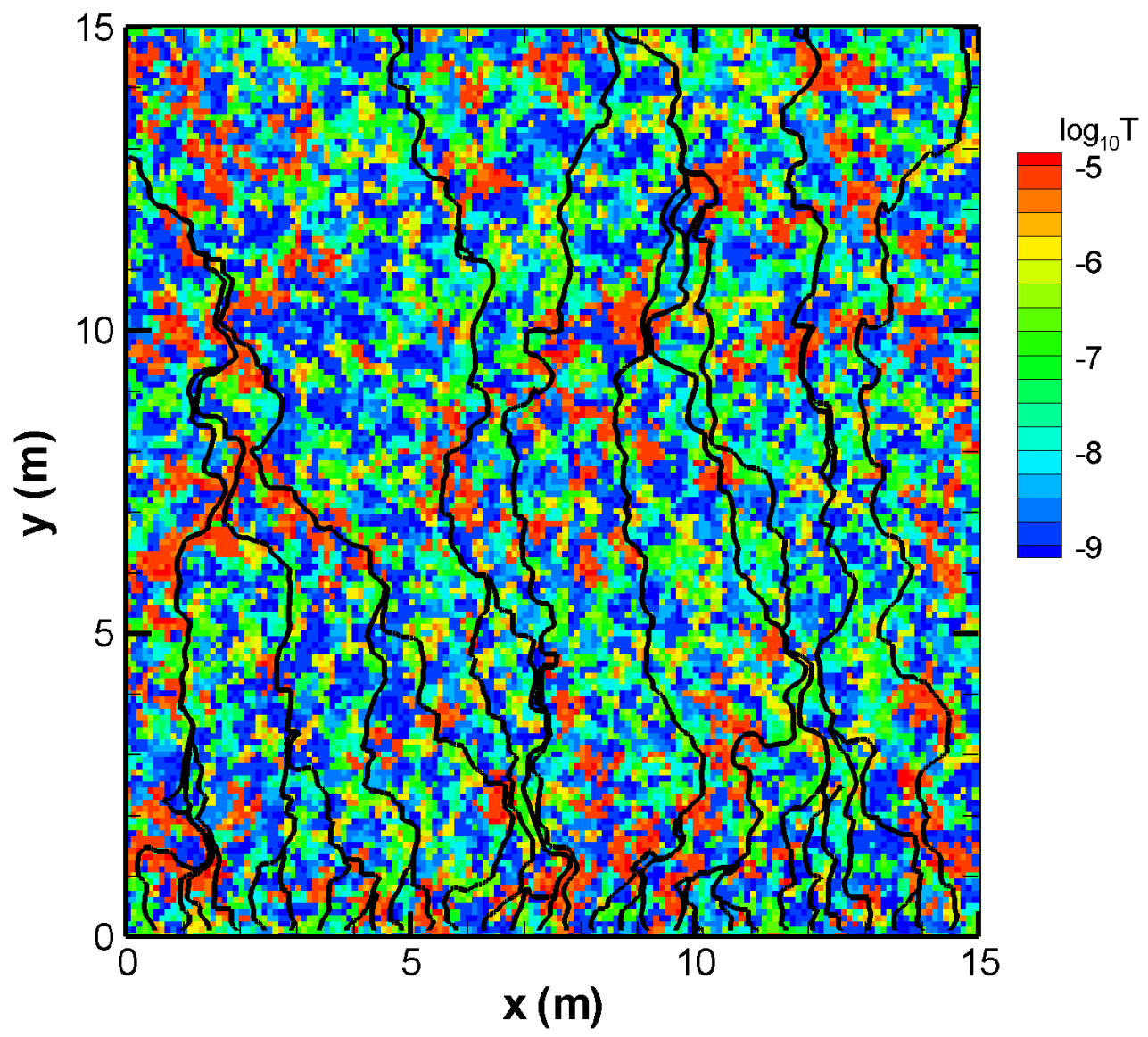

Figure 2. Generated Log transmissivity distribution $\left(T\right.$ in $\left.\mathrm{m}^{2} / \mathrm{s}\right)$, based on six transmissivity groups, the highest of which has a longer correlation length. Stream lines are shown to illustrate the steady-state flow field obtained by imposing a pressure difference between the upper and lower boundaries. 
Figure 3. Calculated breakthrough curves for different types of matrix diffusion and also for different $\alpha$ values. All cases assume probabilities of $20 \%$ small blocks, $30 \%$ intermediate blocks, and 50\% semi-infinite matrix for tracer diffusion at every step. For the "advection-only" curve, diffusion for all matrix types is turned off. For the "single type" curves, matrix diffusion is turned off for all but the one contribution. For the "all types" curves, matrix diffusion is on for all matrix types. The short horizontal bars on the "gouge" curve and the " $\alpha=0.1$ " curve illustrate the spread obtained for five different stochastic realizations of the transmissivity distribution over the fracture plane. 